\title{
Sensorimotor control of speech production: models and data
}

Joseph S. Perkell

Research Laboratory of Electronics, Massachusetts Institute of Technology, USA

https://doi.org/10.36505/ExLing-2006/01/0007/000007

\begin{abstract}
A theoretical, model-based overview of speech production is described in which the goals of phonemic speech movements are in auditory and somatosensory domains and the movements are controlled by a combination of feedback and feedforward mechanisms. Examples of experimental results are presented that provide support for the overview.
\end{abstract}

\section{Introduction}

Speech production is an extraordinarily complex feat of motor coordination that conveys linguistic information, primarily via an acoustic signal, from a speaker to a listener. For information transmission at the phonemic level, speech sounds are differentiated from one another by the type of sound source and also by the wide variety of vocal-tract configurations that are produced by movements of the mandible, pharynx, tongue, soft palate (ve-lum) and lips. These structures are slowly moving; however, because their movements are coarticulated, speech sounds can be produced in intelligible sequences at rates as high as about 15 per second.

The current paper focuses on the control of phonemic movements of the vocal-tract articulators, which are generated by the coordinated contractions of over 50 different muscle pairs. Clearly, the control mechanism is faced with a large number of degrees of freedom and the control problem is im-mensely complicated.

\section{Models and Data \\ Phonemic goals}

It is widely acknowledged that properties of the speech production mechanism have had major influences on the inventories of sounds or phonemes that languages employ, and also on some of the strategies that languages adopt for concatenating phonemes into meaningful sequences. A great deal of research on speech motor control and the mechanisms that underlie sound

ExLing 2006: Proceedings of 1st Tutorial and Research Workshop on Experimental Linguistics, 28-30 August 2006, Athens, Greece 
categories has been directed at identifying the controlled variables, that is, the basic units of speech motor programming. To address this issue, investigators have asked, "What is the task space, or the domain of the fundamental control parameters?"

Our approach to such questions is motivated by observing that the objective of the speaker is to produce sound strings with acoustic cues that can be transformed into intelligible patterns of auditory sensations in the listener. These acoustic cues consist mainly of time-varying patterns of formant frequencies for vowels and glides, and noise bursts, silent intervals, aspiration and frication noises and rapid formant transitions for consonants. The properties of such cues are determined by parameters that can be observed in several domains, including: levels of muscle tension, movements of articulators, changes in the vocal-tract area function and aerodynamic events. Hypothetically, motor control variables could consist of any combination of these parameters.

In order to make the approach to this issue as tractable as possible, we formulate research hypotheses in terms of the function of the DIVA model of speech motor planning (cf. Guenther et al, 2006). DIVA is a neurocomputational model of relations among cortical activity, its motor output and the resulting sensory consequences of producing speech sounds. In the model, phonemic goals are encoded in neural projections (mappings) from premotor cortex to sensory cortex that describe regions in multidimensional auditory-temporal and somatosensory-temporal spaces. The model has two control subsystems, a feedback subsystem and a feedforward subsystem. Feedback control employs error detection and correction to teach, refine and update the feedforward control mechanisms. As speech is acquired and becomes fluent, speech sounds, syllables and words become encoded as strings of feedforward commands.

How are phonemic goal regions determined? One factor is based on properties of speakers' production mechanisms that are characterized by quantal relations between articulation and acoustics (Stevens, 1989). There are a number of examples in which a continuous change in an articulatory parameter produces discontinuous changes in a salient acoustic parameter, resulting in regions of relative acoustic stability and regions of rapid change. Modelling and experimental results support the idea that such regions of stability help to define phonemic goals and sound categories (cf. Stevens, 1989; 1998; Perkell and Nelson, 1985; Perkell et al., 2000).

There are also quantal relations between articulatory movements and the area function, which are expressed when two articulators come into contact with one another. Fujimura and Kakita (1979) have modelled such a "saturation effect" for the vowel /i/ by showing how the vocal-tract cross-sectional area at the acousti- 
cally sensitive place of maximum constriction can be stabilized by pressing the lateral edges of a stiffened tongue blade against the sides of the hard palate.

Another general, model-based principle that likely influences phoneme categories is a balance between sufficient perceptual contrast and ease of articulation (called "economy of effort" - Lindblom, 1990). Other important influences on sound systems are not amenable to being modelled, and it is not claimed that quantal effects and a balance between contrast and economy of effort can themselves account for the wide variety of sounds that are found in different languages. Nevertheless, quantifiable principles can provide a general framework for the formation of sound patterns, and more specific implementations of these particular principles can be utilized by individual speakers. An example of one such implementation is given below for a saturation effect. Other examples below provide support for some of the features of the DIVA model, including the use of sensory goals regions and feedback and feedforward control.
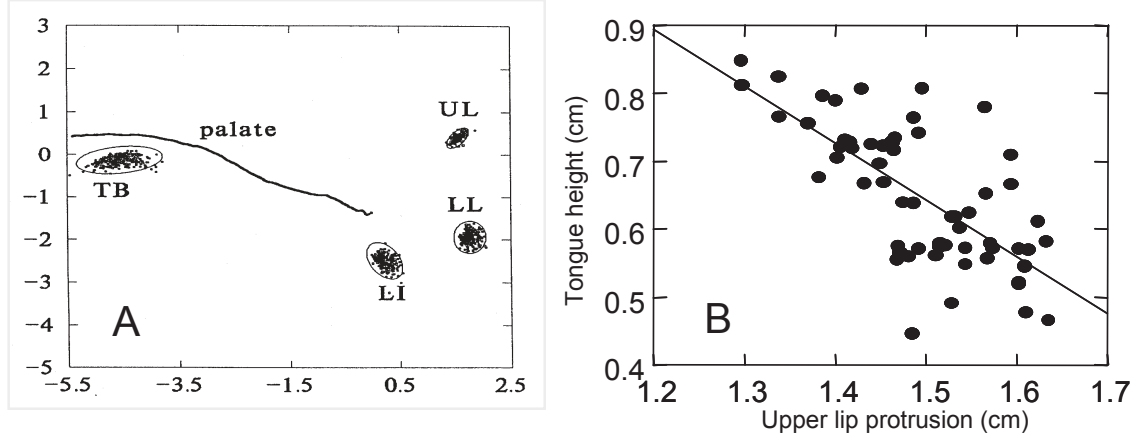

Figure 1. A: Example of points on the tongue body (TB), Upper lip (UL), lower lip (LL) and lower incisors (LI) for many repetitions of the vowel /u/ by a single speaker in a context phrase. B: Tongue height versus lip protrusion for many repetitions of the vowel /u/ by a single speaker.

\section{Auditory goals for /u/ and /r/: Motor equivalence}

The vowel $/ \mathrm{u} /$ in American English is produced by forming a narrow constriction with tongue raising in the velo-palatal region and by rounding the lips. Because of many-to-one relations between vocal-tract shapes and acoustics, approximately the same acoustic output can be produced with more tongue raising and less lip rounding and vice-versa. Figure 1B shows an example of tongue height versus lip protrusion for many repetitions of the vowel $/ \mathrm{u} /$ by a single speaker. The negative correlation reflects a motorequivalent trading relation between the two articulations. Such reciprocal variation of two independently controllable articulations provides evidence 
that the goal for the vowel $/ \mathrm{u} /$ is in an acoustic/auditory frame of reference, rather than a spatial or gestural one (Perkell et al., 1993). Evidence of an acoustic/auditory goal for $/ \mathrm{r} /$ in American English was obtained in a similar motor-equivalence study by Guenther et al. (1999).

\section{Auditory goals: Relations between speech production and perception}

Further insight about auditory goals can be gained by examining relations between speech production and perception. It is well known that that if an individual is born without hearing, that person has a very difficult time learning how to speak intelligibly. On the other hand, if someone acquires speech normally and then becomes profoundly deaf postlingually, that person's speech can remain intelligible for decades without any useful hearing. However, the speech of such individuals does gradually develop some anomalies following hearing loss. A number of studies have been conducted on speakers who became deaf in adulthood, went without hearing for a number of years and then received a cochlear implant. Results have shown that phonemic goals are stable, but contrasts can gradually diminish without hearing. Restoration of some hearing with an implant usually results in parallel improvements in perception, measures of contrast in production and speech intelligibility (cf. Perkell et al., 2000; Vick et al, 2001).

In another approach, we have conducted studies of vowel and sibilant production and perception with 19 normal-hearing young adult speakers of American English. For two vowel contrasts and the sibilant $\left(/ \mathrm{s} / / / \int /\right)$ contrast, we measured each speaker's degree of produced contrast and the speaker's auditory acuity for the contrast. Produced vowel contrast distances were measured in articulatory and formant (F1, F2) spaces and the produced sibilant contrast was measured as the difference in spectral means between $/ \mathrm{s} /$ and $/ \int /$. Auditory acuity was measured as the subjects' ability to discriminate between pairs of natural-sounding synthetic stimuli along continua between each of the contrasting sounds. Both studies found that speakers with greater acuity produced the sounds with greater contrast. To interpret these results, we assume that spoken-language learners find it advantageous to be as intelligible as possible and therefore acquire auditory goal regions that are as distinct as possible. We reason that speakers who can perceive fine acoustic details will learn auditory goal regions that are smaller and spaced further apart than speakers with less acute perception, because the speakers with more acute perception are more likely to reject poorly produced tokens when acquiring the goals (Perkell et al., 2004a, 2004b). 


\section{A somatosensory goal and a saturation effect: The sibilant contrast}

We have hypothesized that the sibilant sound /s/ has a somatosensory goal as well as an auditory one. The somatosensory goal is characterized by a saturation effect, which enhances the contrast of $/ \mathrm{s} /$ with its homologue, $/ \mathrm{S} /$. As schematized in Fig. 2, $/ \mathrm{J} /$ is produced by positioning the tongue blade so that there is a sublingual cavity. This cavity adds volume and complexity to the resonant cavity anterior to the constriction and thereby contributes to the lower spectral center of gravity of the frication noise. On the other hand, /s/ is produced by pressing the under-side of the tongue blade against the lower alveolar ridge and incisors, which eliminates the sublingual cavity and results in a smaller anterior resonator that contributes to a higher spectral center of gravity. When the tongue blade is moved forward to produce an $/ \mathrm{s} /$, once the sublingual cavity is eliminated, further contraction of the muscles that produce the forward movement will increase the contact pressure but will have a negligible effect on the size of the resonant cavity. Thus making this contact, which can be considered an somatosensory goal for the sound $/ \mathrm{s} /$, is characterized as a saturation effect. We also made measurements of the consistency of sublingual contact during /s/ production in the above-described perception/production study. The most distinct sibilant productions were made by subjects who used contact in producing /s/ but not $/ \mathrm{S} /$, and had higher acuity. Subjects who did not use contact differentially and had lower acuity produced the least distinct contrasts. Intermediate degrees of contrast were found with subjects who used contact differentially or had higher acuity (Perkell et al, 2004b).

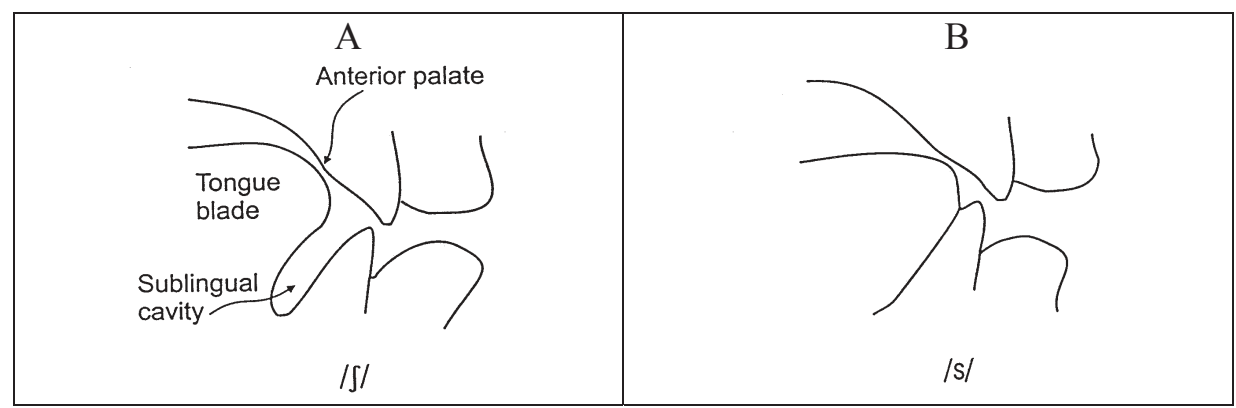

Figure 2. Schematic of tongue blade configurations for producing an $/ \mathrm{J} /$ (A) and an $/ \mathrm{s} /(\mathrm{B}) . / \mathrm{S} /$ is produced with a sublingual cavity, which contributes to the lower mean frequency of its acoustic spectrum; /s/ is produced with contact between the under side of the tongue blade and the lower incisors. 


\section{Feedback and feedforward control}

To learn more about feedback and feedforward control mechanisms in speech, investigators have conducted a large number studies in which auditory or somatosensory feedback (or both) have been perturbed and subjects' compensatory responses have been measured. Some of these studies have used steadystate perturbations, such as inserting a bite block between the teeth or blocking hearing with masking noise; others have used intermittent articulatory or auditory perturbations that the subjects cannot anticipate. Unanticipated perturbations of jaw movements, palatal shape, or auditory feedback have revealed that mechanisms are available that can detect and correct production errors within about 100 to $150 \mathrm{~ms}$ from the onset of the perturbation. Therefore, if a movement lasts long enough, somatosensory and auditory errors can be corrected during the movement itself by closed-loop feedback mechanisms. However, many articulatory movements in fluent speech do not last long enough to be corrected by feedback mechanisms. It follows that fluent adult speech production is controlled almost entirely by feedforward mechanisms, as in the DIVA model.

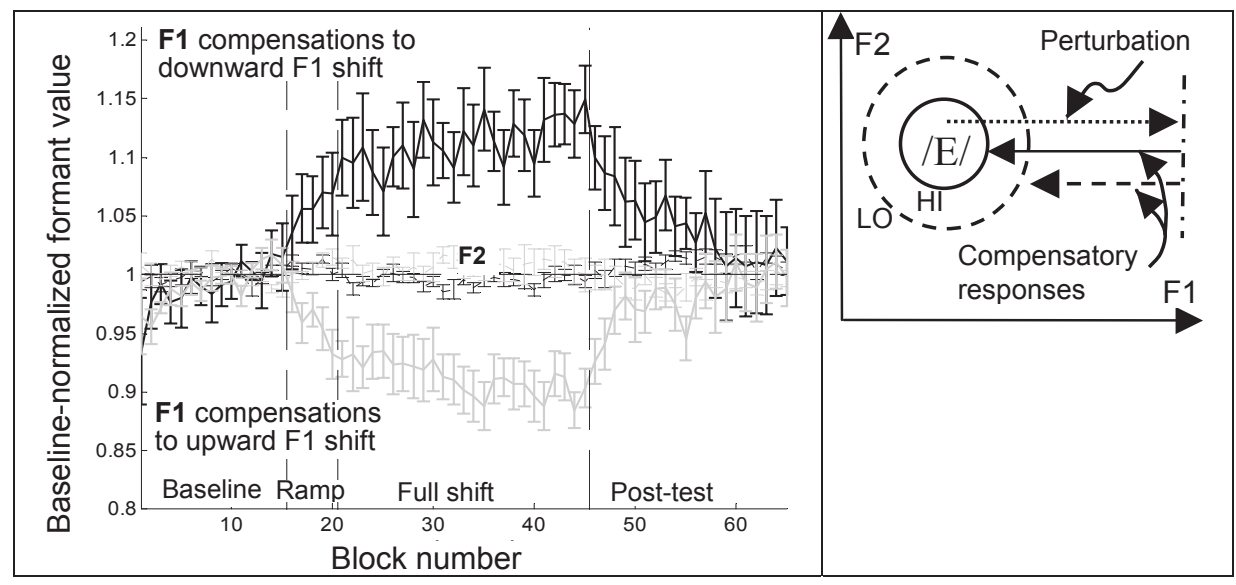

Figure 3. A: Compensatory responses to F1 shifts in normal-Baseline-normalized formant value f subjects' baseline-normalized F1 and F2 vs. block number. Each block contains one repetition of each of 18 different words in the corpus. The curves above baseline show the average of 10 subjects' productions in response to a downward shift of F1; the curves below baseline, the average of 10 subjects' responses to an upward F1 shift. B: Schematic of goal regions and compensatory responses for $/ \varepsilon /$ for a high-acuity speaker (solid circle) and a low-acuity speaker (dashed circle). F1 perturbation is indicated by the dotted arrow, and compensatory responses, by the solid and dashed arrows. 
Figure 3A shows the results of an experiment that investigated feedforward control in 20 normal-hearing speakers. The subjects pronounced $/ \mathrm{C} \varepsilon \mathrm{C} /$ words while the first formant frequency (F1) of the vowel in their auditory feedback being was shifted in nearly real time (18 ms. delay), without their being aware of the shift (Villacorta et al., 2005). Ten of the subjects received upward shifts and the other 10, downward shifts. The plots show that the subjects partially compensated for the shifts over many trials by modifying their productions so that F1 moved in the direction opposite to the shift. The subjects' auditory acuity was also measured. There was a significant correlation between subjects' acuity and amount of compensation to F1 shift: speakers with better acuity tended to compensate more.

What underlies this correlation between acuity and compensation? Figure 3B schematizes how two speakers differing in acuity, and therefore in the sizes of their auditory goal regions for the vowel $/ \varepsilon /$, might respond to a perturbation of $F 1$. The high-acuity speaker has a smaller goal region. The perturbation of F1 is indicated by a dotted arrow pointing to the right, and the shifted value of F1, by a vertical broken line. This high-acuity speaker, in response to the shift in F1, will produce a greater compensatory response (middle arrow) than the one with lesser acuity. This is because the speaker continues to compensate until the F1 of his or her auditory feedback (which includes the shift) moves into the goal region. The distance between the shifted value of F1 (vertical line) and the edge of the goal region is greater for the high acuity speaker. In the DIVA model, auditory feedback provides closed-loop corrections of current motor commands and then modifications of feedforward commands for subsequent movements.

\section{Summary}

According to our theoretical overview and experimental results, the control variables for phonemic movements consist of auditory-temporal and somatosensory-temporal goal regions, which correspond to expected sensory consequences of producing speech sounds. Goal regions for languages in general and for individual speakers are determined by a number of factors, including quantal and saturation effects. Findings of motor-equivalent trading relations for the sounds $/ \mathrm{u} /$ and $/ \mathrm{r} /$ provide evidence that their goals are at least partly auditory. Auditory feedback is crucial for acquisition of phonemic goals, and it is needed to maintain appropriate motor commands with vocal-tract growth and perturbations. The goals are usually stable; however degree of contrast can diminish with prolonged postlingual hearing loss and increase with hearing restored by a cochlear implant. Findings that speakers with better acuity produce more distinct sound contrasts indicate that more acute speakers may learn smaller, more distinct goal regions.

Feedback and feedforward control operate simultaneously; however feedforward control predominates in fluent speech. Frequently used sounds (syllables and words) are encoded as feedforward commands. Feedback control intervenes when a perturbation produces a large enough mismatch between expected and produced sensory 
consequences. In such cases if the movement lasts long enough, a correction is expressed during the movement itself, i.e., closed loop). Otherwise, the correction is incorporated into feedforward control of subsequent movements.

Since the DIVA model is formulated in terms of patterns of cortical connectivity and activity, it can be tested with brain imaging experiments. And, as reflected in the examples described above, it provides a valuable means of quantifying relations among phonemic specifications, brain activity, articulatory movements and the speech sound output.

\section{Acknowledgements}

The work from our laboratory that is described in this chapter was done in collaboration with a number of people, including Frank Guenther, Harlan Lane, Melanie Matthies, Mark Tiede, Majid Zandipour, Margaret Denny, Jennell Vick and Virgilio Villacorta. Support was from grants R01-DC001925 and R01-DC003007 from the National Institute on Deafness and Other Communication Disorders, National Institutes of Health.

\section{References}

Fujimura, O. and Kakita, Y. 1979. Remarks on quantitative description of lingual articulation. In B. Lindblom and S. Öhman (eds.) Frontiers of Speech Communication Research, Academic Press.

Guenther, F.H., Espy-Wilson, C., Boyce, S.E., Matthies, M.L., Zandipour, M. and Perkell, J.S. 1999. Articulatory tradeoffs reduce acoustic variability during American English /r/ production. J. Acoust. Soc. Am., 105, 2854-2865.

Guenther, F.H., Ghosh, S.S., and Tourville, J.A. 2006. Neural modelling and imaging of the cortical interactions underlying syllable production. Brain and Language, 96, 280-301.

Lindblom, B.E.F. 1990. Explaining phonetic variation: A sketch of the H\&H theory. In W.J. Hardcastle and A. Marchal (Eds.), Speech Production and Speech Modelling. (pp. 403-439). Netherlands: Kluwer Academic Publishers.

Perkell, J.S., Guenther, F.H., Lane, H., Matthies, M.L., Perrier, P., Vick, J., Wilhelms-Tricarico, R. and Zandipour, M. 2000. A theory of speech motor control and supporting data from speakers with normal hearing and with profound hearing loss, J. Phonetics 28, 233-372.

Perkell J.S., Guenther F.H., Lane, H., Matthies, M.L., Stockmann, E., Tiede, M. and Zandipour, M. 2004a. The distinctness of speakers' productions of vowel contrasts is related to their discrimination of the contrasts, J. Acoust. Soc Am. 116, 2338-44.

Perkell, J.S., Matthies, M.L., Svirsky, M.A. and Jordan, M.I. 1993. Trading relations between tonguebody raising and lip rounding in production of the vowel $/ \mathrm{u} /$ : A pilot motor equivalence study, J. Acoust. Soc. Am. 93, 2948-2961.

Perkell J.S., Matthies, M.L., Tiede, M., Lane, H., Zandipour, M., Marrone, N., Stockmann, E. and Guenther, F.H. 2004b. The Distinctness of Speakers' /s/-/S/ Contrast is related to their auditory discrimination and use of an articulatory saturation effect, JSLR 47, 1259-69.

Perkell, J.S. and Nelson, W.L. 1985. Variability in production of the vowels /i/ and /a/, J. Acoust. Soc. Am. 77, 1889-1895.

Stevens, K. N. 1989. On the quantal nature of speech. J. Phonetics 17, 3-46.

Stevens, K.N. 1998. Acoustic Phonetics, MIT Press, Cambridge, MA.

Vick, J., Lane, H., Perkell, J.S., Matthies, M.L., Gould, J., and Zandipour, M. 2001. Speech perception, production and intelligibility improvements in vowel-pair contrasts in adults who receive cochlear implants. J. Speech, Language and Hearing Res. 44, 1257-68.

Villacorta, V., Perkell, J.S., and Guenther, F.H. 2005. Relations between speech sensorimotor adaptation and perceptual acuity. J. Acoust. Soc. Am. 117, 2618-19 (A). 